\title{
Langages
}

http://www.necplus.eu/LGA

Additional services for Langages:

Email alerts: $\underline{\text { Click here }}$

Subscriptions: Click here

Commercial reprints: Click here

Terms of use : $\underline{\text { Click here }}$

\section{Pour une linguistique des odeurs : présentation}

\author{
Georges Kleiber et Marcel Vuillaume
}

Langages / Volume 2011 / Issue 181 / March 2011, pp 3 - 15

DOI: 10.3917/lang.181.0003, Published online: 03 April 2013

Link to this article: http://www.necplus.eu/abstract S0458726X11181016

How to cite this article:

Georges Kleiber et Marcel Vuillaume (2011). Pour une linguistique des odeurs : présentation. Langages, 2011, pp 3-15 doi:10.3917/lang.181.0003

Request Permissions : $\underline{\text { Click here }}$ 


\section{Georges Kleiber}

Université de Strasbourg \& LiLPa/Scolia (EA 1339)

Marcel Vuillaume

Université de Nice Sophia Antipolis \& Groupe MOD (Molécules, Olfaction, Discours)

\section{Pour une linguistique des odeurs : présentation}

\section{UN SUJET RELATIVEMENT NOUVEAU EN LINGUISTIQUE}

L'objectif de ce numéro de Langages est d'explorer le territoire linguistique des odeurs. Il ne s'agit pas d'une terra incognita. Psychologues, ethnologues, anthropologues, sociologues, philosophes ${ }^{1}$ et linguistes ont à maintes reprises arpenté ce domaine et y ont ouvert de fructueuses pistes d'investigation (voir infra). Il reste que, si on compare la situation des odeurs à celle des couleurs, avec lesquelles on les associe couramment, force est de reconnaître que les descriptions linguistiques des odeurs ne sont pas légion et que, contrairement aux couleurs pour lesquelles on dispose d'études nourries aussi bien sémantiques (universalistes ou non) que syntaxiques et morphologiques, les odeurs n'ont guère donné lieu à un éventail d'analyses aussi large et diversifié. Il s'agit donc incontestablement d'un sujet qui, même s'il n'est pas totalement inédit, présente néanmoins en linguistique un aspect de nouveauté et d'originalité certain.

\section{UN SUJET ATTRAYANT, STIMULANT, MAIS DIFFICILE}

Il n'est guère besoin de souligner longuement pourquoi les odeurs représentent pour les linguistes, les sémanticiens surtout, un sujet tout particulièrement intéressant. Tous ceux qui ont abordé les odeurs ont observé que leur caractéristique principale était de ne pas donner lieu à des dénominations : il n'y a pas de noms d'odeurs comme il y a des noms de couleurs. Face à des noms de couleur simples, comme le bleu, le rouge, le vert, etc., ou composés, comme le rose saumon, le gris perle, etc., il n'y a pas de noms d'odeur correspondants. Les odeurs " n' ont pas de nom » et l'olfaction est " un sens sans parole » (Howes 1986, cité par Dubois, 2008 : 46), donnant lieu à ce que J. Candau et A. Jeanjean (2006: 51) appellent

1. Vient de paraître une Philosophie de l'odorat (Jaquet 2010), qui propose de substituer le sentir beau au sentir bon. 
joliment un «silence olfactif». Des noms comme puanteur, relents ou encore parfum, effluves peuvent donner à penser le contraire, mais ce sont des noms qui renvoient à des types qualitatifs (d'ordre divers) (cf. Boisson 1997) et non des identifiants d'odeurs, c'est-à-dire que ce ne sont pas des noms qui indiquent de quelle odeur précise il s'agit, comme bleu, par exemple, indique qu'il s'agit de la couleur bleue.

De manière plus générale, toutes les observations font état d'un langage des odeurs beaucoup plus pauvre, plus limité et plus instable, moins systématique que le langage d'autres domaines sensoriels comme le visuel ou l'acoustique :

Ce lexique olfactif [...] est [...] imprécis, (on compte un nombre élevé de descripteurs différents pour un même stimulus), instable, asymétrique et, dans la vie quotidienne, peu élaboré et souvent idiosyncrasique, ce qui a conduit certains chercheurs à qualifier un peu trop vite l'odorat de sens muet. (Candau, $2000: 68)^{2}$

On a aussi fait observer que le vide lexical olfactif n'était sans doute propre qu'à notre civilisation occidentale ${ }^{3}$ et que des langues et cultures dites plus « exotiques » pouvaient posséder des dénominations d'odeurs spécifiques, mais ce point reste sujet à discussion ${ }^{4}$. Quoi qu'il en soit exactement de son universalité, l'absence de noms d'odeurs conduit tout naturellement à se poser deux questions : Pourquoi en va-t-il ainsi ? et Comment dénomme-t-on ou désigne-t-on alors les odeurs?

Ces deux questions, qui correspondent aux deux principales portes d'entrée de la plupart des investigations, linguistiques comme non linguistiques, sur les odeurs, soulignent éloquemment combien les odeurs constituent un sujet passionnant, intrigant pour les linguistes et, au-delà, pour tous ceux qui s'intéressent de près ou de loin au langage. Un sujet attrayant et stimulant, qui ne peut qu'exciter la curiosité et la sagacité passionnée du sémanticien, mais qui se révèle vite difficile, car délicat et complexe à aborder. Les réponses aux deux questions qui servent de point de départ à toutes les escapades dans le domaine des odeurs engagent, en effet, des dimensions explicatives beaucoup moins claires que les questions qui les suscitent.

La première question débouche sur des explications neurophysiologiques, de chimie (cf. les molécules odorantes), sémantiques, anthropologiques et culturelles dont il est difficile de cerner l'exacte importance et le degré de pertinence de chacune d'entre elles, si on n'est pas soi-même spécialiste de chacune d'entre

2. Voir aussi Boisson (1997 : 31) : «Le lexique olfactif ne présente donc pas la belle systématicité du lexique des couleurs, vraisemblablement parce que l'espace olfactif est beaucoup plus complexe ».

3. Rouby \& Sicard (1997 : 69) citent Dupire (1987) qui mentionne que les Seerer N'dut du Sénégal auraient des termes de base pour les odeurs. Dubois (2008 : 47) signale Boisson (1997) et les lexicalisations spécifiques opérées essentiellement dans le domaine des mauvaises odeurs et elle mentionne en note Mouélé (1997) qui a relevé une dizaine de termes spécifiques d'odeurs dans la langue Wanzi du Gabon.

4. Lenclud (2006 : 16) émet ainsi des doutes sur l'existence, mise en avant par Hombert (1992), de noms dénotant des odeurs dans une langue parlée du Gabon. 
elles ${ }^{5}$. Relevons simplement la triple explication qui a pu être avancée dans la littérature psycho-linguistique ${ }^{6}$ :

- Le sens olfactif est pour les humains moins important que les sens visuel et acoustique. Les perceptions visuelle et acoustique constituent, en effet, leur première source d'orientation dans l'espace, alors que l'olfaction se trouve restreinte à des secteurs particuliers, comme la sexualité, l'évaluation de la nourriture (Heeschen 2007), des pratiques religieuses, professionnelles (Candau 2000), etc. Cette restriction serait à la source de la restriction langagière sur les odeurs. M. Plümacher et P. Holz (2007 : 7) notent toutefois que l'on peut tenir également le raisonnement inverse en argumentant que c'est le caractère limité du langage des odeurs qui est à l'origine de la moindre importance du sens olfactif. Et donc, s'il s'agit d'un " sens méconnu », c'est parce que l'homme n'a pas de langage développé à propos des odeurs et non pas tellement parce qu'il a, comme il est souvent dit, « levé le nez » pour regarder et entendre ce qui se passe autour de lui.

- Une deuxième explication, neurophysiologique cette fois-ci, se base sur les structures corticales de traitement de l'information olfactive. L'information olfactive est d'abord traitée dans la partie du cerveau la plus vieille du point de vue de l'évolution, le système limbique, avant que le traitement ne se poursuive dans les parties du néocortex, spécialement dans l'hémisphère droit. Les connexions directes vers le néocortex gauche, où le langage est traité, sont beaucoup moins développées ${ }^{7}$. L'organisation cérébelleuse de la perception d'odeurs ne serait pas, pour cette raison, adéquatement synchronisée avec le traitement du langage. L'absence d'un lexique stable des odeurs en serait la conséquence, selon K. Burdach (1988 : 22), cité par M. Plümacher et P. Holz (2007). De plus, l'implication du système limbique dans l'organisation de fonctions viscérales du corps, à savoir le système nerveux autonome et le développement des émotions, permet d'expliquer le fait que la perception olfactive affecte le comportement humain de façon inconsciente : "For not all odor stimuli may reach a conscious level if they end at the level of the limbic system" (Plümacher \& Holz, $2007: 6)^{8}$.

- La troisième explication, également d'ordre neurophysiologique, est que les stimuli acoustiques et visuels peuvent être gradués (scalarisés) selon la longueur d'onde (wavelength), la teinte (hue), brillance ou luminosité (brightness) ou le volume du bruit. Les odeurs ne peuvent être classifiées selon des propriétés physiques ou chimiques, comme l'ont montré P. Vroon et al. (1996 : 23),

5. Pour en avoir une idée rapide, on peut se reporter aux articles portant sur l'olfaction dans l'ouvrage collectif de Plümacher \& Holz (eds) (2007).

6. Nous nous appuyons ici sur l'introduction de Plümacher \& Holz (2007 : 7-8).

7. Plümacher \& Holz (2007 : 6) citent ici Bear et al. (2001 : 576 sqq.), Vroon et al. (1996 : 25) et Zucco \& Tressoldi (1989).

8. Et ils citent les articles de Zucco (2007) et de Chernigovskaya \& Arshavsky (2007) qui font partie du volume édité. 
cités par M. Plümacher et P. Holz (2007 : 8), qui soulignent que "There is no linear proportional relation between strength of olfactory stimuli and intensity of perception". C'est l'absence de telles relations qui expliquerait l'absence pour les odeurs de catégorisation et de lexicalisation stables.

La seconde question conduit apparemment à une réponse plus sereine et plus consensuelle que la première, puisque tous les commentateurs ont indiqué que le procédé généralement utilisé pour pallier l'absence de noms d'odeurs consiste à recourir au nom de la source de l'odeur (odeur de citron, odeur de poisson, etc.). Les difficultés surgissent toutefois très vite, dès lors que l'on essaie d'attribuer un statut au résultat de ce procédé : $\mathrm{s}^{\prime}$ agit-il de dénomination ${ }^{9}$, de désignation ${ }^{10}$ ? Est-ce qu'en parlant d'une odeur de citron, on utilise une dénomination ou seulement une désignation ? Et, en admettant que l'on ait fait un choix en faveur de l'une ou l'autre, faut-il considérer les variantes comme l'odeur du citron, l'odeur d'un citron, l'odeur de citron, etc., comme ayant le même statut que celui que l'on assigne à une odeur de citron ? Et, si c'est un adjectif comme citronnée ou végétale qui accompagne odeur (cf. une odeur citronnée/végétale), y verra-t-on encore la source de l'odeur ou s'agit-il de désigner par là des propriétés de l'odeur ? Comme s'interroge A. Holley (1997 : 25),

une odeur dite fruitée doit-elle son appartenance à la catégorie du "fruité" parce qu'elle partage des traits perceptifs avec les autres odeurs semblablement qualifiées ou simplement parce qu'elle émane d'un objet appartenant à la catégorie botanique de fruit ?

Bref, s'ouvre ici la difficile et complexe problématique de la diversité des modalités linguistiques de dénomination/désignation et/ou caractérisation ${ }^{11}$ des odeurs.

Le traitement de cette problématique conduit tout naturellement à se poser la question, tout aussi redoutable, de notre conceptualisation des odeurs. Comment concevons-nous ou catégorisons-nous les odeurs (cf. Candau \& Wathelet 2011, ce volume) ? Dit autrement, et peut-être de manière plus révélatrice, quel type d'entités sont pour nous les odeurs ? Plusieurs points s'avèrent ici névralgiques.

- En premier lieu, l'opposition concret (matériel)/abstrait (immatériel). Les positions ne sont pas univoques. Certains optent pour des entités matérielles, d'autres pour l'abstrait ou l'immatériel. Les connaissances scientifiques dont nous disposons actuellement aiguillent plutôt vers l'option « substances matérielles » (molécules odorantes) alors que les considérations ontologiques

9. Une expression est la dénomination d'une entité, si cette entité a eu par convention cette expression comme name, c'est-à-dire si elle a réellement été dénommée ou appelée ainsi (cf. librairie pour le magasin où l'on vend des livres, nager pour une certaine manière de se déplacer dans l'eau et Paul pour le voisin du dessus).

10. On parle de désignation quand l'expression n'a pas été attribuée a priori en propre à l'entité à laquelle elle renvoie, mais permet néanmoins d'y accéder par l'intermédiaire des informations (descriptives ou autres) qu'elle comporte (cf. le magasin où j'étais hier / cette librairie ou le voisin du dessus).

11. Par caractérisation, on entend la description des propriétés ou qualités d'une entité. 
" naïves » peuvent faire pencher la balance du côté de l'abstrait, comme le montre cette définition recueillie par S. David et al. (1997: 54) dans leur enquête sur les définitions des odeurs données par des étudiants : C'est un parfum, quelque chose d'abstrait que l'on peut sentir. Avant d'opérer son choix, il convient, bien entendu, d'examiner les tenants et aboutissants linguistiques de la chose, c'est-à-dire d'étudier de près le comportement linguistique des termes d'odeurs, ce qui n'a été entrepris que dans de rares études. Faut-il le préciser? Le résultat ne se logera pas forcément dans une des deux cases de départ.

- En deuxième lieu, la dimension massif/comptable. Là aussi, l'hésitation est possible et, selon le point de vue adopté, les odeurs seront ou des entités massives ou des entités comptables. Et c'est de nouveau la mise en avant des manifestations linguistiques qui permettra de se faire une idée plus précise $\mathrm{d}^{\prime}$ une situation qui n'est pas aussi homogène que l'on peut le penser. Pourquoi, par exemple, odeur est-il difficilement massifiable alors que cela ne semble pas poser de problèmes pour parfum ?

- En troisième lieu, relativement au statut nominal des noms d'odeurs : s'agitil ou non de noms de substances ou de noms de propriétés, c'est-à-dire de noms comme beurre, eau, etc. ou de noms comme beauté, tristesse, etc. ? Leur dépendance ontologique et dénominative ou désignationnelle vis-à-vis de leur source, ainsi que d'autres facteurs - comme, par exemple, la polysémie de parfum (sens de 'bonne odeur' et sens de 'substance aromatique solide ou liquide') - font que la majeure partie des commentateurs optent clairement pour le statut de propriété ${ }^{12}$ : "In linguistic terms [conclut S. David (2002 : 95) à la fin de son analyse linguistique des termes d'odeurs], this means that the linguistic type for words currently associated with the olfaction realm is one of property". Mais, là encore, le doute est permis, et la prise en compte de leurs propriétés linguistiques ne dirige pas forcément vers leur placement dans le camp des propriétés.

D'autres questions, quoique moins centrales, demandent néanmoins à être résolues si l'on entend cerner le plus complètement possible le statut ontologique des odeurs : qu'en est-il de leur rapport avec le temps, avec l'espace, avec la mémoire, etc. ?

\section{3. ÉTATS DES LIEUX}

S. David et al. (1997 : 52) constataient en 1997 qu'il y avait « peu de recherches sur l'expression linguistique des structures conceptuelles relatives aux odeurs ». Même si J. Candau (2000) a fort justement noté que « le langage des odeurs est en voie d'être sérieusement exploré ", ce constat reste encore aujourd'hui d'actualité. On dispose de plus d'études sur l'olfaction dans les domaines de la chimie, de

12. Voir sur ce point Lenclud (2006). 
la physiologie, de la neuro-physiologie, de la psychologie, de l'ethnologie, de l'anthropologie, etc., que dans celui de la linguistique proprement dite. Les études « linguistiques » sur les odeurs, comme déjà signalé supra, sont en effet, si on les compare à celles qui traitent des couleurs, relativement peu nombreuses. On pourrait jouer sur le titre (Un sens méconnu) du premier chapitre de l'ouvrage de J. Candau (op. cit.) en parlant ludiquement du « sens méconnu d'un sens méconnu ». Les principales études langagières sur les odeurs - menées, pour ce qui est du français, essentiellement par la psychologue D. Dubois et des spécialistes comme C. Rouby, A. Holley, S. David, C. Boisson, etc. - figurent principalement dans des numéros thématiques de revue (numéro 24 de 1997 d'Intellectica, dirigé par D. Dubois et A. Holley ; numéro 47 de Terrain de 2006) et des ouvrages collectifs (Catégorisation et cognition : de la perception au discours, 1997, dirigé par D. Dubois ; Olfaction, Taste and Cognition, 2002, dirigé par C. Rouby et al. ; Speaking of Colors and Odors, Aspects, 2007, dirigé par M. Plümacher et P. Holz ; Le Sentir et le Dire, 2009, dirigé par D. Dubois).

À l'exception de certaines d'entre elles, que nous présenterons infra, ces études langagières $n^{\prime}$ ont pas pour vocation première une description linguistique des odeurs. Leur ambition est de nature plus psycholinguistique que linguistique. La problématique qu'elles traitent est, en effet, celle qui prévaut aussi dans la littérature sur les couleurs : elles cherchent avant tout à voir, dans une perspective cognitiviste affirmée, comment on catégorise, comment on nomme, comment on classe, comment on identifie, comment on décrit et comment on (se) représente les odeurs. Elles ne s'appuient donc pas sur les connaissances scientifiques précises que peuvent fournir les autres disciplines qui s'intéressent à l'olfaction (chimie, neurologie, etc.), même si elles ne rompent pas le pont avec ces sciences. Leur objet principal est la mise au jour de la catégorisation et de la représentation « olfactives » langagières qu'opèrent les sujets parlants. Autrement dit, pour faire écho-écot au plaisant titre de l'article de D. Dubois (2008) ${ }^{13}$, il s'agit de débusquer le sens commun de ce sens commun qu'est l'odorat.

L'absence, du côté des odeurs, de dénominations semblables à celles dont disposent les couleurs ne permet toutefois pas des investigations du type « universaliste » et exclut de ces recherches certaines questions, en particulier celles qui sont au centre de la discussion toujours ouverte sur l'existence ou la nonexistence de couleurs universelles suscitée par l'hypothèse des basic color terms de B. Berlin et P. Kay (1969) ${ }^{14}$. Elles sont conduites en vue de déterminer sur le terrain comment se construit et se déploie la diversité olfactive. Elles essaient ainsi principalement de décrire la façon dont les sujets désignent et caractérisent

13. Le titre de l'article est «Sens communs et sens commun : expériences sensibles ; connaissance(s) ou doxa ? » publié dans la revue Langages 170.

14. Signalons ici l'excellent article de Boisson (1997), déjà cité, qui étudie, sous l'angle de la dénomination, les « variations et régularités linguistiques » du lexique des odeurs dans soixante langues prises dans neuf familles de langues différentes. 
les odeurs qu'ils perçoivent, et recourent à la méthodologie, classique en psychologie cognitive, qui consiste à demander directement au sujet parlant ce qu'est pour lui une odeur, quels types d'odeurs il connaît, etc., comme l'illustrent les deux questions posées à la base de l'enquête dénominative menée dans S. David et al. (1997: 53) :

Selon vous, qu'est-ce qu'une odeur?

Quels types d'odeurs distinguez-vous dans votre environnement?

De telles enquêtes ont conduit à plusieurs types de résultats. Premièrement, elles ont permis d'apporter une réponse langagière à l'absence de dénominations d'odeurs relevée par tous les spécialistes. S'il n'y a pas de "noms d'odeurs », les locuteurs disposent de plusieurs moyens pour exprimer les odeurs. Il y a, en premier lieu, comme déjà mentionné supra, la forme odeur de $X$ avec $X$ comme source de l'odeur, mais on trouve également des adjectifs relationnels formés sur des noms qui indiquent également la source (odeur fruitée = odeur de fruits, odeur florale $=$ odeur de fleurs), des adjectifs qualificatifs indiquant l'effet produit par l'odeur (en -able tel insoutenable, en -ant tel irritant) et des expressions plus complexes (non analysées en détails) comme ça sent la pomme ou l'odeur de l'aprèsrasage de son mari ou encore l'odeur des trottoirs mouillés après la pluie (Dubois, $2008: 46)^{15}$.

En deuxième lieu, elles confirment la prégnance de l'axe dit hédonique (bonnes odeurs/mauvaises odeurs) relevé par tous les commentateurs comme élément principal structurant des types d'odeurs : les locuteurs répartissent en effet habituellement les odeurs en " odeurs qui (leur) sont agréables » et en " odeurs qui (leur) sont désagréables ». Un tel universel axiologique pose directement le problème de la variation interculturelle et intersubjective des jugements évaluatifs à la base du classement hédonique des odeurs.

En troisième lieu, les définitions communes obtenues se révèlent être centrées sur l'expérience subjective, comme le prouvent, d'une part, la présence de nombreuses marques restreignant la validité de la définition ou caractérisation donnée au domaine du locuteur (cf. les cadratifs énonciatifs du type de selon moi, pour moi, je classe les odeurs...) et, d'autre part, la très forte subjectivité qui guide la classification des odeurs (les odeurs de mon environnement/entourage, mes propres odeurs) et qui imprègne l'expérience olfactive (une odeur, c'est quelque chose que je perçois par le nez).

Quatrième et dernier résultat, qui n'est pas le moindre, ces enquêtes langagières sur la définition, la reconnaissance et la représentation des odeurs par les sujets parlants débouchent sur un résultat qui peut surprendre. Le fait que les odeurs sont essentiellement définies par les sujets parlants comme odeur de $X$ conduit «à interpréter l'odeur non comme un objet du monde (comme c'est le cas pour la conceptualisation des objets visuels), mais davantage comme un

15. Voir aussi David et al. (1997) et Dubois (2007). 
effet ${ }^{16}$ du monde sur le sujet connaissant » (Dubois, 2008 : 46-47). Pour les sujets parlants, les odeurs n'auraient donc pas vraiment de réalité ontologique ou, autrement dit, n'existeraient pas réellement, mais ne seraient que des " effets ${ }^{17}$ sur les sujets, suscités par "quelque chose" qui n'est cependant pas le "focus" de la définition » (op. cit. : 49) ${ }^{18}$. Est-il pertinent d'aller jusque là ? Ou, dit autrement, le « sens commun » considère-t-il vraiment que les odeurs ne sont que des effets, et non des objets du monde ? S'agit-il réellement ou non de " catégories » comme les autres ? La question mérite un examen approfondi, auquel est consacrée la contribution de J. Candau et $\mathrm{O}$. Wathelet (2011). On se contentera, pour le moment, d'observer que l'enjeu dépasse le cadre de la sémantique des odeurs : $c^{\prime}$ est le problème du rapport avec le réel du sens en général qui se trouve par là-même posé ${ }^{19}$.

Nous avons déjà annoncé leur existence : il y a des études plus proprement linguistiques du domaine des odeurs, $c^{\prime}$ est-à-dire des études dont le but premier est la caractérisation, la description des expressions linguistiques renvoyant aux odeurs et l'analyse de leur comportement discursif. Elles sont cependant extrêmement rares, étant donné que les problèmes cognitifs de la reconnaissance, de la représentation et de l'identification des odeurs paraissent d'une dimension beaucoup plus grande.

On signalera tout d'abord l'étude de sentir et de sens menée par J.-J. Franckel (2004) (voir Theissen, ce volume), qui apporte des indications précieuses sur le « sentir » olfactif, même si elle dépasse le cadre de l'olfaction, puisqu'elle entend être valide, dans le cadre d'une sémantique des formes schématiques, pour tous les emplois de sentir et de sens.

On mentionnera ensuite, du point de vue de la linguistique générale, l'analyse comparative entreprise par C. Boisson (1997) qui porte sur une soixantaine de langues. Il faut aussi signaler que la vaste enquête de méthode psycholinguistique menée par le groupe de S. David et al. (1997) présente une forte coloration linguistique, due sans doute à la spécialisation linguistique de $\mathrm{S}$. David. Les auteurs distinguent, en effet, dans leur entreprise deux plans d'analyse, un " plan strictement linguistique » (op. cit. : 52) et un plan cognitivo-psychologique. Sur le plan linguistique, ils proposent :

16. Souligné par l'auteur.

17. ibid.

18. De façon plus précise : «Ce travail incite donc à récuser la pertinence de l'exclusivité d'une description "objective" des odorants et à considérer l'odeur comme objet psychologique structuré de manière décisive par des similitudes "expériencées" (plutôt que perçues) par un sujet agissant sur le monde (et non plus contemplatif) et qui tient davantage compte des états de la matière inscrits dans la temporalité que d'une discrétisation du monde en objets immuables. » (Dubois \& Rouby, 1997 : 15)

19. Voir à ce propos le numéro 170 de Langages (2008) sur Discours et sens commun, dirigé par Sarfati, dans lequel est publié l'article de Dubois. Ce numéro montre de façon paradoxale, par le contenu essentiellement constructiviste des articles qu'il contient, que, sur le sens commun, il n'y a décidément pas de sens... commun ! On peut le regretter ou non. 
à partir d'un traitement automatique des données verbales traitées comme corpus, [...] une analyse linguistique des résultats, en tant que contribution pour le français, à la connaissance des odeurs sur le plan des formes linguistiques. (op. cit. : 53)

D'où de nombreux (r)enseignements sur la forme simple ou complexe des dénominations et désignations d'odeurs (sans que la distinction entre dénomination et désignation soit clarifiée), sur la fréquence des termes odeur, parfum, senteur, fragrance, odorat, odorant, parfumé, etc., sur les formes impossibles (odeur de vanille vs. *odeur à vanille/*odeur avec (de la) vanille/*odeur en jasmin) expliquées en recourant aux analyses de P. Cadiot (1997) sur les prépositions à, avec, en et de, sur les paraphrases en avoir (l'odeur de cheval $\rightarrow$ le cheval a une odeur vs. $\rightarrow{ }^{*}$ le cheval est une odeur) pour l'explication desquelles ils s'appuient sur D. Corbin et M. Temple (1994), sur la construction des adjectifs en -é (cf. odeur citronnée), etc.

Certains de ces résultats « linguistiques » sont repris dans S. David (2002), qui constitue incontestablement l'article « le plus linguistique » sur les odeurs en français. L'auteur entend, en effet, décrire la grammaire d'un sous-ensemble de substantifs du français associés à l'olfaction avec deux objectifs :

- identifier, d'un point de vue linguistique, les propriétés de ces mots et examiner à quel degré ils ressemblent et se différencient d'autres modes sensoriels comme la vision (couleurs) et l'audition ;

- évaluer certaines conséquences de l'hypothèse que les expressions linguistiques contribuent à la structuration de la représentation en mémoire (op. cit. : 82).

En se fondant sur les propriétés argumentales et les propriétés sortales, considérées comme les corrélats grammaticaux des propriétés référentielles (c'est-à-dire des propriétés d'un objet dans le monde), S. David essaie de mettre en relief les principaux aspects sémantiques des substantifs analysés. Elle relève ainsi, en premier lieu, l'hétérogénéité de ces substantifs, en montrant qu'ils ne présentent pas tous les mêmes propriétés (morphologiques, constructionnelles). Elle décrit, tout particulièrement, la bi-typicité de parfum qui, contrairement à odeur (qui ne correspond, selon elle, qu'au type 'propriété'), répond aussi au type 'substance' comme eau, fer, or, rejoignant ainsi l'analyse polysémique de parfum des dictionnaires. En deuxième lieu, elle analyse la nature de la relation entre une odeur et sa source et conclut à la non autonomie de la première par rapport à la seconde : "French terms associated with the olfactory field, and the term odeur in particular, refer to entities that cannot be separated from their sources" (op. cit. : 91). Elle prolonge son investigation en démontrant que la source ne peut être considérée comme une partie de l'odeur et que ni odeur ni parfum, effluve, fragrance et senteur, à la différence de puanteur et exhalaison, n'ont de propriétés temporelles. Les différentes analyses opérées convergent vers un même résultat déjà mentionné supra: les substantifs d'odeurs sont des noms de propriété et parfum "has two different types, property and material object" (op. cit. : 95) (voir Schnedecker, ce volume). S. David rejoint par là-même, à la fin de son article (ibid.), les conclusions psycho-linguistiques de D. Dubois, à savoir que la non 
autonomie des noms d'odeurs a pour conséquence des classifications des odeurs fondées sur la ressemblance avec leur source et non dissociées du sujet percevant et que, d'un point de vue cognitif, les entités nommées par odeur n'ont pas le caractère objectif des entités visuelles et auditives, mais doivent être considérées simplement comme des effets, "i.e., as manifestations of a sensation that cannot be dissociated from the subject sensing it)" (ibid.).

\section{OBJECTIF}

Tout en conservant des liens avec les autres champs d'étude (cf. la contribution de Candau \& Wathelet, ce volume), ce numéro de Langages, comme l'indique clairement le titre choisi, se place résolument sur le terrain linguistique arpenté par S. David (2002). Non pas que nous jugions inutiles ou secondaires les entreprises de type psycho-linguistique présentées supra : ces analyses ont mis au jour des aspects fondamentaux de notre appréhension et représentation des odeurs et il ne s'agit donc nullement de les tenir pour partie négligeable. Mais, elles ont tendance à extrapoler sur le plan linguistique des résultats dont la validité et la pertinence se limitent au seul plan qui les a produits. Certaines de leurs conclusions, notamment celles qui concernent la problématique « linguistique » que constitue la dénomination des odeurs, ne nous semblent ainsi pas totalement légitimes d'un point de vue linguistique, parce qu'elles résultent d'une confusion entre désignation et dénomination. Il est nécessaire de reprendre le débat avec des arguments plus linguistiques, sur ce point, comme sur d'autres, telle la question de la « nature » des odeurs. Considérer les odeurs comme n'étant que des effets ressentis par un sujet parlant est un résultat qui peut sembler spectaculaire, mais est-il vraiment légitime ? Il n'est pas sûr que, sous l'angle de leur expression linguistique, donc pour les sujets parlants, les odeurs apparaissent effectivement comme étant des effets. Un des objectifs principaux de certains articles de ce numéro sera précisément de caractériser au moyen de critères linguistiques et de méthodes diverses leur profil sémantique et d'apporter ainsi une réponse à la question ontologique qu'elles posent.

Dans cette perspective, le numéro essaiera tout particulièrement de reprendre certains résultats linguistiques obtenus par S. David (2002), que nous venons d'exposer supra. S'agit-il vraiment de noms de propriétés ? Et, si ce ne sont pas des noms de ce type, de quoi s'agit-il alors ? La réponse à cette question constituera un des apports principaux de ce numéro et, parce qu'elle s'écarte des vues classiques en ce domaine, puisqu'elle défendra un statut différent de celui de noms de propriétés, contribuera fortement à l'originalité de l'entreprise. Celleci s'exprimera également à travers une autre problématique essentielle pour les odeurs, celle de la caractérisation de leurs propriétés. Cette problématique sera examinée sous des angles différents qui permettront de faire ressortir, en même temps que la complexité de la question, des points de stabilité et des zones d'instabilité. 


\section{PRÉSENTATION DES CONTRIBUTIONS}

Georges Kleiber et Marcel Vuillaume (Sémantique des odeurs) ont choisi d'arpenter le terrain ontologico-sémantique : en s'appuyant sur des propriétés linguistiques des termes d'odeurs, ils tentent de déterminer le type d'entités que sont pour nous, sujets parlants, les odeurs. Partant de l'opposition abstrait/concret envisagée dans ses différentes conceptions, ils sont conduits à infirmer la thèse classique des noms d'odeurs comme noms de propriétés. Les odeurs sont des entités irréductibles et les noms qui les dénomment tels odeur, parfum, etc., le sont donc également : ils ne se laissent ranger ni avec les noms des matières ou de substances, ni avec les noms de propriétés, mais forment une catégorie irréductible correspondant à la spécificité du sens qui les fait exister pour nous, à savoir l'olfaction.

Joël Candau et Oliver Wathelet (Les catégories d'odeurs en sont-elles vraiment ?) abordent les odeurs sous l'angle de leur catégorisation : leur enquête menée sur la base de données ethnographiques met en avant qu'il s'agit d'une catégorisation "relâchée ", ni classique ni de caractère prototypique : les odeurs catégorisées sont celles qui produisent un effet (intensité et valeur hédonique), celles qui sont familières ou que l'on ne connaît pas, celles que l'on pense utiles, celles qui ont une propriété intrinsèque identifiable, etc.

Peter Blumenthal ( Odeur » - profils combinatoires), dans une perspective de linguistique de corpus et à l'aide de la théorie des profils combinatoires, s'attache à dégager la « logique » du nom odeur et de ses synonymes depuis le $\mathrm{XVI}^{\mathrm{e}}$ siècle. Il montre ainsi que la composante la plus constante de leur " potentiel catégoriel » est la prégnance de la dimension formation/sensation, qui connaît des variations, selon les époques, marquées par le recul de senteur au profit d'odeur et des prédicats verbaux et par prédominance au $\mathrm{XX}^{\mathrm{e}}$ siècle de l'aspect de la sensation sur celui de la formation.

Hélène Vassiliadou et Marie Lammert ( Odeurs » et dimensions hédonique à travers le prisme des adjectifs) se proposent de relever, de classer et d'analyser les adjectifs avec lesquels se combine le nom odeur pour accéder au cœur du sens de ce terme et, corollairement, confirmer (ou infirmer) certaines des conclusions obtenues par d'autres chemins dans la littérature sur les odeurs et mettre en relief de nouvelles propriétés liées à leur caractérisation et à leur catégorisation.

Catherine Schnedecker (Quand la sémantique se met au(x) parfum(s)) consacre son étude à un nom d'odeur plus spécifique qu'odeur, le nom parfum. L'examen sémantique approfondi de ce terme a pour premier résultat de mettre en relief, via des propriétés morpho-syntaxiques, deux nouveaux emplois de parfum, qui s'ajoutent aux deux habituellement recensés par les dictionnaires. En second lieu, il apporte, à partir d'une analyse de corpus détaillée, un éclairage comparatif révélateur sur la dynamique qualitative interne de l'opposition parfum-'odeur' et parfum-'substance'. 
Anne Theissen ("Sentir » : les constructions prédicatives de l'olfaction) a choisi d'aborder les odeurs par l'intermédiaire des constructions verbales comportant le verbe sentir en emploi olfactif. La description des différents types de constructions verbales, les « extériorisées » comme les "intériorisées », conduit, par l'intermédiaire des contraintes et l'analyse des constituants impliqués, à d'intéressantes observations sur la sémantique des odeurs tout entière.

Irène Tamba (Odeurs, exclamation et haut degré) se penche sur deux types de constructions exclamatives verbales comportant un prédicat verbal d'olfaction (ça pue! et Qu'est-ce que ça pue!) pour étudier leur dimension intensive. Elle se propose d'examiner ce qu'apporte l'exclamation simple ou en qu'est-ce à l'expression de l'intensité d'une sensation olfactive.

\section{Références}

BEAR M.-F. et al. (2001), Neuroscience. Exploring the Brain, Baltimore: Williams and Wilkins.

BerLIN B. \& KAY P. (1969), Basic Color Terms. Their Universality and Evolution, Berkeley: University of California Press.

Boısson C. (1997), "La dénomination des odeurs : variation et régularités linguistiques ", Intellectica 24, 29-49.

BURDACH K. (1988), Geschmack und Geruch : Gustatorische, olfaktorische und trigeminale Wahrnehmung, Bern : Huber.

CADIOT P. (1997), Les prépositions abstraites en français, Paris : Armand Colin.

CAndau J. (2000), Mémoires et expériences olfactives. Anthropologie d'un savoir-faire sensoriel, Paris : Presses Universitaires de France.

CANDAu J. \& JeAnjean A. (2006), " Des odeurs à ne pas regarder ", Terrain 47, 51-68.

CANDAU J. \& WATHELET O. (2011), "Les catégories d'odeurs en sont-elles vraiment ? ", Langages 181, Paris : Armand Colin (ce volume).

CHERNIGOVSKAYA T. \& ARSHAVSKY V. (2007), "Olfactory and visual processing and verbalization: Cross-cultural and neurosemiotic dimensions", in M. Plümacher \& P. Holz (eds), Speaking of Colors and Odors, Aspects, Amsterdam: John Benjamins Publishing Company, 227-238.

Corbin D. \& TEMPLE M. (1994), "Le monde des mots et des sens construits ", Cahiers de lexicologie 65, 5-28.

DAVID S. (2002), “Linguistic Expressions for Odors in French”, in C. Rouby et al. (eds), Olfaction, Taste, and Cognition, Cambridge: Cambridge University Press, 82-99.

DAVID S. et al. (1997), " L'expression des odeurs en français : analyse lexicale et représentation cognitive ", Intellectica 24, 51-83.

Dubols D. (éd.) (1997), Catégorisation et cognition : de la perception au discours, Paris : Kimé.

Duboıs D. (éd.) (2009), Le Sentir et le Dire, Paris : L'Harmattan.

DuBoIs D. (2007), “From psychophysics to semiophysics: Categories as acts of meaning. A case study from olfaction and audition, back to colors", in M. Plümacher \& P. Holz (eds), Speaking of Colors and Odors, Aspects, Amsterdam: John Benjamins Publishing Company, 67-184.

DuBoIs D. (2008), "Sens communs et sens commun : expériences sensibles ; connaissance(s) ou doxa ? ", Langages 170, 41-53. 
Dubols D. \& Holley A. (éds) (1997), Intellectica $\mathrm{n}^{\circ} 24$ : Olfaction : du linguistique au neurone, Saint Denis : MSH Paris-Nord.

Dubois D. \& Rouby C. (1997), "Une approche de l'olfaction : du linguistique au neuronal ", Intellectica 24, 9-20.

DUPIRE M. (1987), " Des goûts et des odeurs : classification et universaux ", L'Homme 104, 5-25.

FRANCKEL J.-J. (2004), " Sentir / sens ", Linx 50, 103-134.

HEESCHEN V. (2007), “Attractivness and adornment: Reference to colors and smells in Papuan speech communities", in M. Plümacher \& P. Holz (eds), Speaking of Colors and Odors, Aspects, Amsterdam: John Benjamins Publishing Company, 85-112.

HOLLEY A. (1997), "Le physiologiste et la catégorisation des odeurs ", Intellectica 24, 21-27.

Hombert J.-M. (1992), "Terminologie des odeurs dans quelques langues du Gabon ", Pholia 7 , 61-65.

HowES D. (1986), "Le sens sans parole : vers une anthropologie de l'odorat ", Anthropologie et Sociétés 10, 29-46.

JAQUET C. (2010), Philosophie de l'odorat, Paris : Presses Universitaires de France.

LENCLUD G. (2006), " La nature des odeurs (remarques) ", Terrain 47, 5-18.

MouÉLÉ M. (1997), "L'apprentissage des odeurs chez les Waanzi : note de recherche ", Enfance 1, 209-222.

PlÜMACHER M. \& Holz P. (2007), "Speaking of Colors and Odors", in M. Plümacher \& P. Holz (eds), Speaking of Colors and Odors, Aspects, Amsterdam: John Benjamins Publishing Company, 1-17.

Plümacher M. \& Holz P. (eds) (2007), Speaking of Colors and Odors, Aspects, Amsterdam: John Benjamins Publishing Company.

Rouby C. \& SiCaRD G. (1997), " Des catégories d'odeurs ? ", in D. Dubois (éd.), Catégorisation et cognition : de la perception au discours, Paris : Kimé, 59-81.

Rouby C. et al. (eds) (2002), Olfaction, Taste, and Cognition, Cambridge: Cambridge University Press.

SARfATI G.-E. (dir.) (2008), Langages ${ }^{\circ} 170$ : Discours et sens commun, Paris : Armand Colin.

SCHNEDECKER C. (2011), "Quand la sémantique se met au parfum ", Langages 181, Paris : Armand Colin (ce volume).

TERRAIN 47 : Odeurs, septembre 2006, Ministère de la culture / Maison des sciences de I'Homme.

THEISSEN A. (2011), "Sentir : les constructions prédicatives de l'olfaction ", Langages 181, Paris : Armand Colin (ce volume).

VROon P et al. (1996), Psychologie der Düfte, Zürich: Kreuz.

Zucco G. M. (2007), “Odor memory: the unique nature of a memory system”, in M. Plümacher \& P. Holz (eds), Speaking of Colors and Odors, Aspects, Amsterdam: John Benjamins Publishing Company, 155-165.

Zucco G. M. \& TRESSOLDI P. E. (1989), “Hemispheric differences in odour recognition”, Cortex 25, 607-615. 